\title{
Points entiers sur les courbes hyperelliptiques
}

\author{
par
}

\author{
Dimitrios Poulakis (Théssalonique)
}

1. Introduction. Soient $k$ un corps de nombres et $A$ son anneau des entiers. On notera $\bar{k}$ une clôture algébrique de $k$. Soit $F$ un polynôme absolument irréductible de $k[X, Y]$ tel que la courbe $F(X, Y)=0$ soit de genre non nul. On sait, d'après Siegel [8], qu'il n'existe qu'un nombre fini de couples $(x, y) \in A \times A$ tels que $F(x, y)=0$. La méthode de Siegel ne permet pas de déterminer de façon effective ces couples.

Dans le cas où $\mathbb{F} \in \mathbb{Z}[X, Y]$ et le genre de la courbe $F(X, Y)=0$ est 1 , Baker et Coates [1] ont donné un majorant de la hauteur $\max \{|x|,|y|\}$ des points entiers de la courbe, explicitement calculable en fonction des coefficients de $F$. Schmidt [4] a récemment amélioré ce résultat de Baker et Coates, et l'a étendu à un corps de nombres $k$ quelconque.

Dans ce travail on s'intéresse aux majorations de la hauteur des points entiers sur des courbes hyperelliptiques. Rappelons qu'une courbe $C$ sur $k$ est dite hyperelliptique si son corps de fonctions $\bar{k}(C)$ contient une fonction $f$ de degré 2 ([9]); il en résulte que $\bar{k}(C)$ possède un $\bar{k}(f)$-automorphisme $\tau$ tel que $\tau \neq \operatorname{Id}$ et $\tau^{2}=$ Id. Supposons maintenant que la courbe $C$ définie par $F(X, Y)=0$ soit hyperelliptique de genre $g \geq 2$. Notons $\Sigma$ l'ensemble des anneaux de valuation discrète de $\bar{k}(C)$ qui contiennent $\bar{k}$ et $\Sigma_{\infty}$ l'ensemble des éléments de $\Sigma$ qui se trouvent au-dessus de l'anneau de valuation discrète de $\bar{k}(X)$ définie par $1 / X$.

Considérons l'ensemble $V(\mathbb{Q})$ de valeurs absolues de $\mathbb{Q}$, qui contient la valeur absolue ordinaire et pour tout premier $p \in \mathbb{Z}$ la valeur absolue $|\cdot|_{p}$ définie par $\left|p^{n} a / b\right|_{p}=p^{-n}$ pour $a, b \in \mathbb{Z}$ avec $(a, b)=1$. Soit $V(k)=\left\{|\cdot|_{v}\right.$ : $v \in M(k)\}$ l'ensemble des valeurs absolues de $k$ qui prolongent les éléments de $V(\mathbb{Q})$. Si $\boldsymbol{x}=\left(x_{0}, \ldots, x_{n}\right)$ est un point de l'espace projectif $\boldsymbol{P}^{n}(k)$ et $v \in M(k)$, on note $|\boldsymbol{x}|_{v}=\max \left\{\left|x_{0}\right|_{v}, \ldots,\left|x_{n}\right|_{v}\right\}$. On appelle la quantité

$$
H_{k}(\boldsymbol{x})=\prod_{v \in M(k)}|\boldsymbol{x}|_{v}^{d_{v}}
$$

où $d_{v}$ sont les degrés locaux, hauteur de $\boldsymbol{x}$ (relativement au corps de 
nombres $k$ ) et la quantité

$$
H(\boldsymbol{x})=H_{k}(\boldsymbol{x})^{1 / d}
$$

où $d$ est le degré de $k$, hauteur absolue de $\boldsymbol{x}([7])$. Si $f \in k\left[X_{1}, \ldots, X_{n}\right]$ on définit $|f|_{v}, H_{k}(f)$ et $H(f)$ en termes du vecteur des coefficients de $f$. Aussi quand $a \in k$ on note $H_{k}(a)=H_{k}((1, a))$.

Dans [7], chap. 8, J.-P. Serre montre que s'il existe $V \in \Sigma_{\infty}$ tel que $\tau(V) \in \Sigma_{\infty}$, le problème de la recherche d'un majorant effectif pour la hauteur des couples $(x, y) \in A \times A$ qui vérifient $F(x, y)=0$ peut être réduit à l'application de la méthode de Baker. Dans ce travail on calcule un tel majorant. Plus précisément, on montre le résultat suivant :

ThÉorÈme. Soient $N$ le degré total de $F$ et $D_{k}$ le discriminant de $k$. On suppose qu'il existe $V \in \Sigma_{\infty}$ tel que $\tau(V) \in \Sigma_{\infty}$. Alors si $(x, y) \in A \times A$ vérifie $F(x, y)=0$ on a

$$
\max \left\{H_{k}(x), H_{k}(y)\right\}<\exp \left\{c(d, N) W^{3 \cdot 10^{5} N^{3} 3^{2 g} d^{2} g^{2 g+3}}\right\}
$$

où

$$
c(d, N)<(3 N)^{d^{3} 2^{N^{2}} N^{5 N^{2}}} \quad \text { et } \quad W=\left|D_{k}\right| H_{k}(F)^{2 \cdot 10^{4} 3^{g} N^{15}} .
$$

EXEMPLES. 1. Soit $F$ un polynôme absolument irréductible de $k[X, Y]$ tel que $F=F_{g+2}+F_{g+1}+F_{g}$ où $F_{i}$ est un polynôme homogène de degré $i$ et $g \geq 2$. On suppose que la courbe $C$ définie par l'équation $F(X, Y)=0$ n'a pas d'autres singularités que le point $(0,0)$ qui est un point multiple ordinaire. Alors on vérifie facilement que $C$ est une courbe hyperelliptique de genre $g$.

L'automorphisme $\tau$ de $\bar{k}(C)$ est donné par l'application

$$
\begin{gathered}
X \rightarrow-X F_{g}(X, Y) /\left(F_{g+1}(X, Y)+F_{g}(X, Y)\right), \\
Y \rightarrow-Y F_{g}(X, Y) /\left(F_{g+1}(X, Y)+F_{g}(X, Y)\right) .
\end{gathered}
$$

Si $F_{g+1}=0$ on a $\tau(V)=V$ pour tout $V \in \Sigma_{\infty}$; aussi si les polynômes $F_{g+2}(X, 1), F_{g+1}(X, 1)$ ont une racine en commun, il existe $V \in \Sigma_{\infty}$ tel que $\tau(V)=V$; par conséquent on peut appliquer le théorème. Dans les autres cas la condition du théorème n'est pas satisfaite.

2. Soit $C$ la courbe définie par l'équation

$$
Y^{2 n+2}=a_{0} X^{n+2}+a_{1} X^{n+1} Y^{2}+\ldots+a_{n} X^{2} Y^{2 n}
$$

où $n \geq 5, a_{i} \in k$. On suppose que les racines du polynôme $a_{0} X^{n}+a_{1} X^{n-1}+$ $\ldots+a_{n}$ sont deux-à-deux distinctes. L'application $X \rightarrow Y / X=W, Y \rightarrow$ $X / Y^{2}=T$ définit une application birationnelle de la courbe $C$ sur la courbe définie par l'équation

$$
W^{2}=a_{0} T^{n}+a_{1} T^{n-1}+\ldots+a_{n} .
$$


Donc $C$ est une courbe hyperelliptique de genre $g=[(n-1) / 2] \geq 2$. Les points à l'infini de $C$ sont les points

$$
[x, y, z]= \begin{cases}{[1,0,0],\left[1, \pm \sqrt{a_{n}}, 0\right]} & \text { si } a_{n} \neq 0 \\ {[1,0,0],\left[1, \pm \sqrt{a_{n-1}}, 0\right],\left[1, \pm i \sqrt{a_{n-1}}, 0\right]} & \text { si } a_{n}=0\end{cases}
$$

(en coordonnés homogènes). Sauf $[1,0,0]$, ce sont des points non singuliers. Notons $\tau$ l'automorphisme de $\bar{k}(C)$ défini par l'application $X \rightarrow X, Y \rightarrow$ $-Y$. Dans le cas où $a_{n} \neq 0$, notons $V_{+}, V_{-}$les éléments de $\Sigma_{\infty}$ qui sont associés aux points $\left[1, \pm \sqrt{a_{n}}, 0\right]$; alors $\tau\left(V_{+}\right)=V_{-}$. Dans le cas où $a_{n}=0$ notons $V_{+}, V_{-}, V_{+}^{\prime}, V_{-}^{\prime}$ les éléments de $\Sigma_{\infty}$ qui sont associés aux points $\left[1, \pm \sqrt{a_{n}}, 0\right],\left[1, \pm i \sqrt{a_{n}}, 0\right]$ respectivement. On a $\tau\left(V_{+}\right)=V_{-}$et $\tau\left(V_{+}^{\prime}\right)=V_{-}^{\prime}$. Dans tous les cas on peut donc appliquer le théorème.

Nous utilisons les résultats de [5] et [6] et le théorème de Riemann-Roch pour construire une courbe birationnelle à $F(X, Y)=0$ d'équation $Y_{1}^{2}=$ $G\left(X_{1}\right)$ telle que les coefficients de $G\left(X_{1}\right)$ soient des entiers algébriques d'un corps de nombres fixé $K$ et que les solutions de $F(X, Y)=0$ en entiers de $k$ soient déterminées par les solutions de $Y_{1}^{2}=G\left(X_{1}\right)$ en entiers de $K$. Une majoration de la taille des solutions entières de l'équation hyperelliptique ([3], théorème 1) nous permet d'obtenir notre résultat.

2. Lemmes auxiliaires. Soit $u \in \Sigma$. On note $\operatorname{ord}_{u}(f)$ l'ordre d'une fonction $f \in \bar{k}(C)$ en $u$.

Lemme 1. Soit $V \in \Sigma$. Alors

(i) si $\tau(V)=V$, il existe $f \in \bar{k}(C)$ tel que $\operatorname{ord}_{V}(f)=-2$ et $\operatorname{ord}_{u}(f) \geq 0$ pour tout $u \in \Sigma-\{V\}$,

(ii) si $\tau(V) \neq V$, il existe $f \in \bar{k}(C)$ tel que $\operatorname{ord}_{V}(f)=-1, \operatorname{ord}_{\tau(V)}(f)=$ -1 et $\operatorname{ord}_{u}(f) \geq 0$ pour tout $u \in \Sigma-\{V, \tau(V)\}$.

Démonstration. Soit $L$ le sous-corps de $\bar{k}(C)$ fixé par $\tau$. Alors il existe $h \in \bar{k}(C)$ tel que $L=\bar{k}(h)$. Si $\operatorname{ord}_{V}(h) \geq 0$ et si $t$ est un paramètre local en $V$ on a $h=a_{0}+a_{1} t+\ldots$ Donc $h^{\prime}=h-a_{0} \in V$ et $h^{\prime}$ est un paramètre local en $V \cap L$. De même si $\operatorname{ord}_{V}(h)<0$, alors $h^{\prime}=1 / h$ est un paramètre local en $V \cap L$. Par conséquent, si $\tau(V)=V$ on a $\operatorname{ord}_{V}\left(h^{\prime}\right)=2$, tandis que si $\tau(V) \neq V$ on a $\operatorname{ord}_{V}\left(h^{\prime}\right)=1$ et $\operatorname{ord}_{\tau(V)}\left(h^{\prime}\right)=1$. Posons $f=1 / h^{\prime}$. Comme $[\bar{k}(C): \bar{k}(f)]=2$, la fonction $f$ n'a pas d'autres pôles que $V$ et $\tau(V)$.

Soient $u_{1}, \ldots, u_{r} \in \Sigma$ et $\mu_{1}, \ldots, \mu_{r} \in \mathbb{Z}$; au diviseur $D=\sum_{i=1}^{r} \mu_{i} u_{i}$ est associé le $\bar{k}$-espace linéaire suivant :

$$
\begin{aligned}
L(D)=\left\{h \in \bar{k}(C) \mid \operatorname{ord}_{u_{i}}(h) \geq\right. & -\mu_{i}, i=1, \ldots, r, \text { et } \\
& \left.\operatorname{ord}_{u}(h) \geq 0 \forall u \in \Sigma-\left\{u_{1}, \ldots, u_{r}\right\}\right\} .
\end{aligned}
$$

On note $l(D)$ sa dimension. 
Lemme 2. Posons $E=2 V$ si $V=\tau(V)$ et $E=V+\tau(V)$ si $V \neq \tau(V)$. Alors $l(\mu E)=\mu+1$ pour $\mu=0,1, \ldots, g-1$.

Démonstration. D'après le lemme 1 il existe $f \in \bar{k}(C)-\bar{k}$ tel que $f \in L(E)$. Alors les fonctions $1, f, \ldots, f^{\mu} \in L(\mu E)$, où $\mu \in \mathbb{N}, 1 \leq \mu \leq g-1$, sont $\bar{k}$-linéairement indépendantes. Il en résulte que $l(\mu E) \geq \mu+1$. En particulier $l((g-1) E) \geq g$.

D'autre part, le théorème de Riemann-Roch montre que $l((g-1) E+V)$ $=g$. On a donc $l((g-1) E)=g$ et $L((g-1) E)=L((g-1) E+V)$. Cela entraîne que les fonctions $1, f, \ldots, f^{g-1}$ constituent une base de $L((g-1) E)$. Alors les fonctions $1, f, \ldots, f^{\mu}$ forment une base de $L(\mu E)$, d'où le résultat.

Soient $v \in M(k)$ et $x$ un réel positif. Notons

$$
v(x)= \begin{cases}x & \text { si }|\cdot|_{v} \text { est archimédienne, } \\ 1 & \text { sinon. }\end{cases}
$$

Lemme 3. Soient $n \in \mathbb{N}$ et $u_{i j} \in k(1 \leq i, j \leq n)$ avec $\left|u_{i j}\right|_{v} \leq A$ pour tout $v \in M(k)$. Si le système

$$
\sum_{j=1}^{n} u_{i j} X_{j}=0 \quad(i=1, \ldots, n)
$$

a une solution non triviale, alors il existe une solution $x_{1}, \ldots, x_{n} \in k$ telle que

$$
\left|x_{j}\right|_{v} \leq A^{n-1} v((n-1) !) \quad(j=1, \ldots, n)
$$

pour tout $v \in M(k)$.

Dém onstration. La démonstration est la même que celle du lemme 2 de [2].

DÉFInitions. On appelle $k$-système une famille $\left\{A_{v}\right\}_{v \in M(k)}$ de nombres réels $\geq 1$ indexée par $M(k)$, tels que $A_{v}=1$ presque pour tout $v \in M(k)$ et $A_{v}$ est un élément du groupe des valeurs de $|\cdot|_{v}$ pour tout $v \in M(k)$. On appelle norme du $k$-système $\left\{A_{v}\right\}_{v \in M(k)}$ la quantité

$$
N_{k}\left\{A_{v}\right\}=\prod_{v \in M(k)} A_{v}^{d_{v}} .
$$

Lemme 4. Soit $F(X, Y) \in k[X, Y]$ de degré n en $Y$ et de degré total $N$. On suppose que F n'a pas de facteur multiple de degré positif en $Y$. Soit

$$
Z=a_{0}+a_{1} X+\ldots
$$

une série telle que $F(X, Z)=0$. Alors

(i) Le corps $K=k\left(a_{0}, a_{1}, \ldots\right)$ engendré sur $k$ par les coefficients de $Z$ est un corps de nombres et $[K: k] \leq n$.

(ii) $K=k\left(a_{0}, a_{1}, \ldots, a_{2 n^{2}}\right)$. 
(iii) Il existe un $k$-système $\left\{A_{v}\right\}_{v \in M(k)}$ tel que

$$
\left|a_{s}\right|_{v} \leq A_{v}^{N+s} \quad(s=0,1,2, \ldots)
$$

pour tout $v \in M(k)$ et chaque extension de $|\cdot|_{v}$ sur $K$. On a aussi

$$
N_{k}\left\{A_{v}\right\}<\left(2^{14} N^{6}\right)^{8 N^{3} d} H_{k}(F)^{8 n^{2} N} .
$$

(iv) $S i D_{K}$ est le discriminant de $K$ on a

$$
\left|D_{K}\right|<N^{N d}\left(2^{14} N^{6}\right)^{48 d N^{7}}\left|D_{k}\right|^{n} H_{k}(F)^{48 n^{5} N^{2}} .
$$

Pour une démonstration de ce résultat on peut consulter [4], §5.

3. Construction d'une équation hyperelliptique. Soient $N$ le degré total de $F(X, Y)$ et $n$ le degré de $F(X, Y)$ en $Y$. On suppose que $X$ est une variable et $\Phi$ une fonction algébrique telle que $F(X, \Phi)=0$.

Soient $V \in \Sigma_{\infty}$ tel que $\tau(V) \in \Sigma_{\infty}$, e l'indice de ramification de $V$ et $X_{V}=(1 / X)^{1 / e}$. Alors

$$
\Phi=\sum_{s=s_{0}}^{\infty} a_{s} X_{V}^{s} .
$$

La fonction $\Phi$ a un pôle d'ordre $\leq N e$ en $V$; on a donc $s_{0} \geq-N e$. En ajoutant des coefficients nuls on peut poser $s_{0}=-N e$.

Posons

$$
\bar{\Phi}=X_{V}^{N e} \Phi=a_{s_{0}}+a_{s_{0}+1} X_{V}+\ldots
$$

Il en résulte

$$
X_{V}^{N e n} F\left(X_{V}^{-e}, X_{V}^{-N e} \bar{\Phi}\right)=0 .
$$

Le polynôme $F^{\prime}(U, W)=U^{N e(n+1)} F\left(U^{-e}, U^{-N e} W\right)$ est sans facteur multiple et de degré positif en $W$. Son degré en $W$ est $n$ et son degré total est $\leq n N^{2}$. Alors, d'après le lemme 4 , on voit que le corps engendré par les $a_{i}$, $\bar{K}=k\left(a_{s_{0}}, a_{s_{0}+1}, \ldots\right)$, est un corps de nombres, de degré $[K: k] \leq n$ et de discriminant majoré par

$$
\left|D_{K}\right|<(2 N)^{865 d N^{21}}\left|D_{k}\right|^{n} H_{k}(F)^{48 N^{11}} .
$$

Supposons d'abord que $\tau(V)=V$. D'après le théorème de RiemannRoch, l'espace $L((2 g+1) V)$ est de dimension $g+2$. Le théorème A2 de [6] entraîne qu'il existe des entiers $\pi_{1}, \ldots, \pi_{t} \geq 0$ tels qu'une base de $L((2 g+$ 1) $V$ ) soit de la forme

$$
X^{h} g_{i} \quad\left(1 \leq i \leq t, 0 \leq h \leq \pi_{i}\right) .
$$

Comme le diviseur $(2 g+1) V$ est rationnel sur $K$, on déduit du théorème B2 de [6] que $g_{1}, \ldots, g_{t} \in K(X, \Phi)$. D'après le théorème C2 de [6] le 
développement de $g_{i}$ en $V$ est

$$
g_{i}=\sum_{s=-(2 g+1)}^{\infty} a_{i s} X_{V}^{s}
$$

avec $a_{i s} \in K$; de plus il existe des $K$-systèmes $\left\{A_{v}(V)\right\}$ et $\left\{B_{v}(i)\right\}$, définis pour tout $v \in M(K)$, tels que

$$
\left|a_{i s}\right|_{v} \leq A_{v}(V)^{s+4 N^{3}} B_{v}(i)
$$

et

$$
\begin{aligned}
N_{K}\left\{A_{v}(V)\right\} & <\left(2^{7} N^{5} H(F)\right)^{9 N^{5} \operatorname{deg} K}, \\
N_{K}\left\{B_{v}(i)\right\} & <\left(9 N^{4} H(F)\right)^{365 N^{11} \operatorname{deg} K} .
\end{aligned}
$$

Notons $f_{1}, \ldots, f_{g+2}$ la base de l'espace $L((2 g+1) V)$ et écrivons

$$
f_{i}=\sum_{s=-(2 g+1)}^{\infty} b_{i s} X_{V}^{s} \quad(i=1, \ldots, g+2) .
$$

On a $b_{i s}=a_{i, s+k e}$, où $k \leq g+1$. Alors

$$
\left|b_{i s}\right|_{v} \leq A_{v}(V)^{s+4 N^{3}+(g+1) n} B_{v} \quad(i=1, \ldots, g+2)
$$

où $B_{v}$ est le produit des $B_{v}(i)$.

D'après le théorème de Riemann-Roch on a $l(2 g V)=g+1$. Donc il existe $i$ tel que $b_{i,-2 g-1} \neq 0$. Soit $b_{1,-2 g-1} \neq 0$; alors $\operatorname{ord}_{V}\left(f_{1}\right)=-2 g-1$ et $\operatorname{ord}_{u}\left(f_{1}\right) \geq 0$ pour tout $u \in \Sigma-\{V\}$.

Considérons les fonctions

$$
w_{1}=b_{1,-2 g-1} f_{2}-b_{2,-2 g-1} f_{1}, \ldots, w_{g+1}=b_{1,-2 g-1} f_{g+1}-b_{g+1,-2 g-1} f_{1} .
$$

Il est facile de vérifier que $w_{1}, \ldots, w_{g+1}$ constituent une base de l'espace $L(2 g V)$. Soient

$$
w_{i}=\sum_{s=-2 g}^{\infty} w_{i s} X_{V}^{s} \quad(i=1, \ldots, g+1) .
$$

Alors

$$
\left|w_{i s}\right|_{v} \leq A_{v}(V)^{s+8 N^{3}+2(g+1) n} B_{v}^{2} v(2) .
$$

Comme $l((2 g-1) V)=g$, en utilisant le même procédé on obtient une base $\left\{t_{1}, \ldots, t_{g}\right\}$ de l'espace $L((2 g-1) V)$. Le développement de $t_{i}$ en $V$ est

$$
t_{i}=\sum_{s=-2 g+1}^{\infty} t_{i s} X_{V}^{s} \quad(i=1, \ldots, g)
$$

avec

$$
\left|t_{i s}\right|_{v} \leq A_{v}(V)^{s+16 N^{3}+4(g+1) n} B_{v}^{4} v(4) .
$$


Le lemme 2 entraîne

$$
\begin{aligned}
\bar{k} & =L(V) \varsubsetneqq L(2 V)=L(3 V) \varsubsetneqq \ldots \varsubsetneqq L(2 k V) \\
& =L((2 k+1) V) \varsubsetneqq \cdots \varsubsetneqq L((2 g-2) V)=L((2 g-1) V) .
\end{aligned}
$$

Par suite, en utilisant successivement la méthode précédente on obtient une base $\left\{h_{1}, h_{2}\right\}$ de $L(2 V)$. Le développement de $h_{i}$ en $V$ est

$$
h_{i}=\sum_{s=-2}^{\infty} h_{i s} X_{V}^{s} \quad(i=1,2)
$$

avec

$$
\left|h_{i s}\right|_{v} \leq A_{v}(V)^{s+2^{g}(g+1) n+2^{g+2} N^{3}} B_{v}^{2^{g}} v\left(2^{g}\right) .
$$

Sans restreindre la généralité on peut supposer que $h_{1,-2} \neq 0 ; \operatorname{donc} \operatorname{ord}_{V}\left(h_{1}\right)$ $=-2$ et $\operatorname{ord}_{u}\left(h_{1}\right) \geq 0$ pour tout $u \in \Sigma-\{V\}$.

Les fonctions

$$
\begin{gathered}
l_{0}=1, \quad l_{1}=h_{1}, \quad l_{2}=h_{1}^{2}, \quad \ldots, \quad l_{2 g+1}=h_{1}^{2 g+1}, \\
l_{2 g+2}=f_{1}, \quad l_{2 g+3}=f_{1}^{2}, \quad l_{2 g+4}=f_{1} h_{1}, \quad \ldots, \quad l_{3 g+3}=f_{1} h_{1}^{g}
\end{gathered}
$$

sont des éléments de l'espace $L((4 g+2) V)$ qui est de dimension $3 g+3$. Donc elles sont $\bar{K}$-linéairement dépendantes.

Le développement de $l_{i}$ en $V$ est

$$
l_{i}=\sum_{s=-4 g-2}^{\infty} l_{i s} X_{V}^{s} \quad(i=0, \ldots, 3 g+3) .
$$

Comme $(N-1)(N-2) \geq 2 g$ il en résulte que $N^{3} \geq 2(g+1) n$; donc

$$
2^{g}(g+1) n+2^{g+2} N^{3} \leq 9 \cdot 2^{g-1} N^{3} .
$$

On obtient facilement

$$
\left|l_{i s}\right|_{v} \leq A_{v}(V)^{s+9(2 g+1) 2^{g-1} N^{3}} B_{v}^{2^{g}(2 g+1)} v\left(2^{g(2 g+1)}(s+4 g+3)^{2 g}\right) .
$$

La dépendance linéaire des fonctions $l_{i}(i=1, \ldots, 3 g+4)$ entraîne que le système

$$
\sum_{j=0}^{3 g+3} x_{j} l_{j k}=0 \quad(-4 g+2 \leq k \leq 0)
$$

a une solution non triviale dans $K$. Il résulte alors du lemme 3 qu'il existe une solution du système non triviale $x_{0}, \ldots, x_{3 g+3} \in K$ telle que

$$
\begin{aligned}
\left|x_{j}\right|_{v} \leq A_{v}(V)^{27(g+1)(2 g+1) 2^{g-1} N^{3}} B_{v}^{3(g+1)(2 g+1) 2^{g}} & \\
& \times v\left(2^{3 g(2 g+1)(g+1)}(4 g+3)^{6 g(g+1)}(3 g+3) !\right) .
\end{aligned}
$$


La fonction $\sum_{j=0}^{3 g+3} x_{j} l_{j}$ n'a pas de pôles et s'annule en $V$; donc

$$
\sum_{j=0}^{3 g+3} x_{j} l_{j}=0
$$

Si $x_{2 g+3}=0$, les fonctions

$x_{0}+x_{1} h_{1}+\ldots+x_{2 g+1} h_{1}^{2 g+1}$ et $\quad x_{2 g+2} f_{1}+x_{2 g+4} f_{1} h_{1}+\ldots+x_{3 g+3} f_{1} h_{1}^{g}$, si elles ne sont pas nulles, ont pour valuation en $V, 4 g+2$ et $4 g+1$ respectivement; c'est impossible. Donc $x_{2 g+3} \neq 0$.

Posons

$$
X^{\prime}=h_{1}, \quad \Phi^{\prime}=2 x_{2 g+3} f_{1}+x_{2 g+2}+\sum_{j=1}^{g} x_{2 g+3+j} h_{1}^{j} .
$$

Il résulte de (3.4) que

$$
\begin{aligned}
\Phi^{\prime 2}= & x_{2 g+2}^{2}-4 x_{2 g+3} \sum_{j=0}^{g} x_{j} X^{\prime j}+\sum_{j=1}^{g} x_{2 g+3+j}^{2} X^{\prime 2 j} \\
& +2 x_{2 g+2} \sum_{j=1}^{g} x_{2 g+3+j} X^{\prime j}+2 x_{2 g+4} \sum_{j=2}^{g} x_{2 g+3+j} X^{\prime j+1} \\
& +2 x_{2 g+5} \sum_{j=3}^{g} x_{2 g+3+j} X^{\prime j+2}+\ldots+2 x_{3 g+2} x_{3 g+3} X^{\prime 2 g-1} .
\end{aligned}
$$

Alors on a

$$
\Phi^{\prime 2}=a_{2 g+1}^{\prime} X^{\prime 2 g+1}+a_{2 g}^{\prime} X^{\prime 2 g}+\ldots+a_{0}^{\prime}
$$

où

$$
\begin{aligned}
a_{0}^{\prime} & =x_{2 g+2}^{2}-4 x_{2 g+3} x_{0} \\
a_{2 \lambda+1}^{\prime} & =-4 x_{2 g+3} x_{2 \lambda-1}+2 x_{2 g+2} x_{2 g+2 \lambda+2}+2 \sum_{j=\lambda}^{\mu} x_{2 g+3+j} x_{2 g+2 \lambda+2-j}
\end{aligned}
$$

avec $\mu=2 \lambda-2$ si $2 \lambda-1 \leq g$ et $\mu=g$ si $2 \lambda-1>g$, et

$$
\begin{aligned}
a_{2 \lambda}^{\prime}= & -4 x_{2 g+3} x_{2 \lambda}+x_{2 g+3+\lambda}^{2}+2 x_{2 g+2} x_{2 g+3+2 \lambda} \\
& +2 \sum_{j=\lambda+1}^{\mu} x_{2 g+3+j} x_{2 g+3+2 \lambda-j}
\end{aligned}
$$

avec $\mu=2 \lambda-1$ si $2 \lambda \leq g$ et $\mu=g$ si $2 \lambda>g(\lambda=1, \ldots, g+1)$.

Supposons que le polynôme

$$
E\left(X^{\prime}\right)=a_{2 g+1}^{\prime} X^{\prime 2 g+1}+a_{2 g}^{\prime} X^{\prime 2 g}+\ldots+a_{0}^{\prime}
$$


ait une racine multiple; alors si $\varrho_{1}=\varrho_{2}, \ldots, \varrho_{2 g+1}$ sont ses racines on a

$$
\left(\Phi /\left(X^{\prime}-\varrho_{1}\right)\right)^{2}=a_{2 g+1}^{\prime}\left(X^{\prime}-\varrho_{3}\right) \ldots\left(X^{\prime}-\varrho_{2 g+1}\right) .
$$

Les fonctions $X^{\prime}-\varrho_{i}(i=3, \ldots, 2 g+1)$ ont un seul pôle en $V$; il en résulte que $\Phi^{\prime} /\left(X^{\prime}-\varrho_{1}\right)$ possède un seul pôle en $V$. De plus ord $\operatorname{ord}_{V}\left(\Phi^{\prime} /\left(X^{\prime}-\varrho_{1}\right)\right)=$ $-2 g+1$. D'après la démonstration du lemme 2 on a

$$
L((2 g-1) V)=L((2 g-2) V) .
$$

Comme $\Phi^{\prime} /\left(X^{\prime}-\varrho_{1}\right) \in L((2 g-1) V)$ et $\Phi^{\prime} /\left(X^{\prime}-\varrho_{1}\right) \notin L((2 g-2) V)$ on obtient une contradiction. Donc les racines de $E\left(X^{\prime}\right)$ sont deux-à-deux distinctes.

Les coefficients de $E\left(X^{\prime}\right): a_{0}^{\prime}, a_{1}^{\prime}, \ldots, a_{2 g+1}^{\prime}$ sont des éléments de $K$; on a aussi

$$
\left|a_{0}^{\prime}\right|_{v}, \ldots,\left|a_{2 g+1}^{\prime}\right|_{v} \leq A_{v}(V)^{54(g+1)(2 g+1) 2^{g-1} N^{3}} B_{v}^{6(g+1) 2^{g}(2 g+1)} v\left(2^{7(2 g+1)^{3}}\right) .
$$

Supposons maintenant que $\tau(V) \neq V$. Posons $V_{1}=V$ et $V_{2}=\tau(V)$. Soit $e_{i}$ l'indice de ramification de $V_{i}$; notons $X_{V_{i}}=(1 / X)^{1 / e_{i}}(i=1,2)$. On vérifie, sans peine, que le diviseur $(g+1) V_{1}+(g+1) V_{2}$ est rationnel sur $K$. Il résulte comme précédemment qu'il existe des fonctions $f_{1}, \ldots, f_{g+3} \in$ $K(X, \Phi)$ qui constituent une base de l'espace $L\left((g+1) V_{1}+(g+1) V_{2}\right)$. Le développement de $f_{i}$ en $V_{j}$ est

$$
f_{i}=\sum_{s=-g-1}^{\infty} b_{i s j} X_{V_{j}}^{s} \quad(i=1, \ldots, s, j=1,2) .
$$

Il y a des $K$-systèmes $\left\{A_{v}\left(V_{j}\right)\right\}$ et $\left\{B_{v}(i)\right\}(j=1,2, i=1, \ldots, g+3)$ tels que

$$
\left|b_{i s j}\right|_{v} \leq A_{v}\left(V_{j}\right)^{s+4 N^{3}+(g+1) n} B_{v}
$$

où $B_{v}$ est le produit des $B_{v}(i)$, et

$$
\begin{aligned}
N_{K}\left\{A_{v}\left(V_{j}\right)\right\} & <\left(2^{7} N^{5} H(F)\right)^{9 N^{5} \operatorname{deg} K}, \\
N_{K}\left\{B_{v}(i)\right\} & <\left(9 N^{4} H(F)\right)^{365 N^{11} \operatorname{deg} K} .
\end{aligned}
$$

D'après le théorème de Riemann-Roch on a

$$
l\left(g V_{1}+(g+1) V_{2}\right)=l\left((g+1) V_{1}+g V_{2}\right)=g+2 .
$$

Donc il existe $i_{1}, i_{2}$ tels que $b_{i_{1},-g-1,1} \neq 0$ et $b_{i_{2},-g-1,2} \neq 0$. Soit $b_{i_{1},-g-1,2}$ $=b_{i_{2},-g-1,1}=0$. Alors on a

$$
\operatorname{ord}_{V_{1}}\left(f_{i_{1}}+f_{i_{2}}\right)=-(g+1) \quad \text { et } \quad \operatorname{ord}_{V_{2}}\left(f_{i_{1}}+f_{i_{2}}\right)=-(g+1) .
$$

On remplace ensuite dans la base l'élément $f_{i_{1}}$ par $f_{i_{1}}+f_{i_{2}}$. Alors quitte à multiplier le majorant de $\left|b_{i s j}\right|_{v}$ par $v(2)$ on peut supposer, sans restreindre la généralité, que $b_{1,-g-1,1} \neq 0$ et $b_{1,-g-1,2} \neq 0$. Donc $\operatorname{ord}_{V_{1}}\left(f_{1}\right)=$ $\operatorname{ord}_{V_{2}}\left(f_{1}\right)=-g-1$ et $\operatorname{ord}_{u}\left(f_{1}\right) \geq 0$ pour tout $u \in \Sigma-\left\{V_{1}, V_{2}\right\}$. 
Procédant comme dans le cas précédent et compte tenu du fait que le lemme 2 implique

$$
\begin{gathered}
\bar{k}=L\left(V_{1}\right)=L\left(V_{2}\right) \varsubsetneqq L\left(V_{1}+V_{2}\right)=L\left(2 V_{1}+V_{2}\right)=L\left(V_{1}+2 V_{2}\right) \varsubsetneqq \ldots \\
\ldots \varsubsetneqq L\left((g-1) V_{1}+(g-1) V_{2}\right)=L\left(g V_{1}+(g-1) V_{2}\right)=L\left((g-1) V_{1}+g V_{2}\right)
\end{gathered}
$$

on obtient une base $\left\{h_{1}, h_{2}\right\}$ de $L\left(V_{1}+V_{2}\right)$ telle que le développement de $h_{i}$ en $V_{j}$ soit

$$
h_{i}=\sum_{s=-1}^{\infty} h_{i s j} X_{V_{j}}^{s}
$$

avec

$$
\left|h_{i s j}\right|_{v} \leq A_{v}^{s+2^{g+1}(g+1) n+2^{g+3} N^{3}} B_{v}^{2^{g+1}} v\left(2^{g+2}\right)
$$

où $A_{v}=\max \left\{A_{v}\left(V_{1}\right), A_{v}\left(V_{2}\right)\right\}$. On peut prendre $h_{1,-1,1} \neq 0$ et $h_{1,-1,2} \neq 0$. Donc $\operatorname{ord}_{V_{1}}\left(h_{1}\right)=\operatorname{ord}_{V_{2}}\left(h_{1}\right)=-1$ et $\operatorname{ord}_{u}\left(f_{1}\right) \geq 0$ pour tout $u \in \Sigma-$ $\left\{V_{1}, V_{2}\right\}$.

On considère les fonctions

$$
\begin{gathered}
l_{0}=1, \quad l_{1}=h_{1}, \quad \ldots, \quad l_{2 g+2}=h_{1}^{2 g+2}, \quad l_{2 g+3}=f_{1}, \quad l_{2 g+4}=f_{1}^{2}, \\
l_{2 g+5}=f_{1} h_{1}, \quad \ldots, \quad l_{3 g+5}=f_{1} h_{1}^{g+1} .
\end{gathered}
$$

Le développement de $l_{i}$ en $V_{j}$ est

$$
l_{i}=\sum_{s=-2 g+2}^{\infty} l_{i s j} X_{V_{j}}^{s} \quad(i=1, \ldots, 3 g+5, j=1,2)
$$

et on a

$$
\left|l_{i s j}\right| \leq A_{v}\left(V_{j}\right)^{s+18(g+1) 2^{g} N^{3}} B_{v}^{2^{g+2}(g+1)} v\left(4^{(g+2)^{2}}(s+2 g+2)^{2 g+1}\right) .
$$

Les fonctions $l_{i}$ appartiennent à l'espace $L\left((2 g+2) V_{1}+(2 g+2) V_{2}\right)$ dont la dimension est $l\left((2 g+2) V_{1}+(2 g+2) V_{2}\right)=3 g+5$. Donc ces fonctions $l_{i}(i=0,1, \ldots, 3 g+5)$ sont $\bar{k}$-linéairement dépendantes. Par conséquent le système

$$
\sum_{i=0}^{3 g+5} x_{i} l_{i s 1}=0, \quad \sum_{i=0}^{3 g+5} x_{i} l_{i s 2}=0 \quad(s=-2 g-2, \ldots, 0)
$$

possède une solution non triviale dans $K$. Alors le lemme 3 implique qu'il existe une solution du système non triviale $x_{0}, x_{1}, \ldots, x_{3 g+5} \in K$ telle que

$$
\begin{aligned}
\left|x_{j}\right|_{v} \leq A_{v}^{18(3 g+4)(g+1) 2^{g}} N^{3} B_{v}^{2^{g+2}(g+1)(3 g+4)} & \\
& \times v\left(4^{(g+2)^{2}(3 g+4)}(2 g+2)^{(2 g+1)(3 g+4)}(3 g+4) !\right) .
\end{aligned}
$$

Il en résulte que $\sum_{i=0}^{3 g+5} x_{i} l_{i}=0$ avec $x_{2 g+4} \neq 0$. 
On pose

$$
X^{\prime}=h_{1}, \quad \Phi^{\prime}=2 x_{2 g+4} f_{1}+x_{2 g+3}+\sum_{j=1}^{g+1} x_{2 g+4+j} h_{1}^{j}
$$

et on obtient l'équation

$$
\Phi^{\prime 2}=a_{2 g+2}^{\prime} X^{\prime 2 g+2}+a_{2 g+1}^{\prime} X^{\prime 2 g+1}+\ldots+a_{0}^{\prime}
$$

où

$$
\begin{aligned}
a_{0}^{\prime} & =x_{2 g+3}^{2}-4 x_{2 g+4} x_{0} \\
a_{2 \lambda-1}^{\prime} & =-4 x_{2 g+4} x_{2 \lambda-1}+2 x_{2 g+3} x_{2 g+3+2 \lambda}+2 \sum_{j=\lambda}^{\mu} x_{2 g+4+j} x_{2 g+4+2 \lambda-j}
\end{aligned}
$$

avec $\mu=2 \lambda-2$ si $2 \lambda-1<g+1$ et $\mu=g+1$ si $2 \lambda-1>g+1$, et

$a_{2 \lambda}^{\prime}=-4_{2 g+4} x_{2 \lambda}+x_{2 g+4+\lambda}^{2}+2 x_{2 g+3} x_{2 g+4+2 \lambda}+2 \sum_{j=\lambda+1}^{\mu} x_{2 g+4+j} x_{2 g+4+2 \lambda-j}$

avec $\mu=2 \lambda-1$ si $2 \lambda \leq g+1$ et $\mu=g+1$ si $2 \lambda>g+1(\lambda=1, \ldots, g+1)$.

Supposons que le polynôme

$$
E\left(X^{\prime}\right)=a_{2 g+2}^{\prime} X^{\prime 2 g+2}+a_{2 g+1}^{\prime} X^{\prime 2 g+1}+\ldots+a_{0}^{\prime}
$$

ait une racine multiple; alors si $\varrho_{1}=\varrho_{2}, \varrho_{3}, \ldots, \varrho_{2 g+2}$ sont ses racines on a

$$
\left(\Phi^{\prime} /\left(X^{\prime}-\varrho_{1}\right)\right)^{2}=a_{2 g+2}^{\prime}\left(X^{\prime}-\varrho_{3}\right) \ldots\left(X^{\prime}-\varrho_{2 g+2}\right) .
$$

Il en résulte que les seuls pôles de $\Phi^{\prime} /\left(X^{\prime}-\varrho_{1}\right)$ se trouvent en $V_{1}$ et $V_{2}$ et on a

$$
\operatorname{ord}_{V_{j}}\left(\Phi^{\prime} /\left(X^{\prime}-\varrho_{1}\right)\right)=-g \quad(j=1,2) .
$$

Comme $\operatorname{ord}_{V_{j}}\left(X^{\prime}-\varrho_{1}\right)=-1(j=1,2), \operatorname{ord}_{u}\left(X^{\prime}-\varrho_{1}\right) \geq 0$ pour tout $u \in \Sigma-\left\{V_{1}, V_{2}\right\}$ et $l\left(g V_{1}+g V_{2}\right)=g+1$ on en déduit que les fonctions

$$
1, X^{\prime}-\varrho_{1}, \ldots,\left(X^{\prime}-\varrho_{1}\right)^{g}
$$

constituent une base de l'espace $L\left(g V_{1}+g V_{2}\right)$. Par conséquent il existe $c_{0}, c_{1}, \ldots, c_{g} \in \bar{k}$ tels que

$$
\Phi^{\prime} /\left(X^{\prime}-\varrho_{1}\right)=c_{0}+c_{1}\left(X^{\prime}-\varrho_{1}\right)+\ldots+c_{g}\left(X^{\prime}-\varrho_{1}\right)^{g} .
$$

D'autre part, soit $M$ le sous-espace de $L\left((g+1) V_{1}+(g+1) V_{2}\right)$ engendré par $1, h_{1}, \ldots, h_{1}^{g+1}$. Comme $l\left((g+1) V_{1}+(g+1) V_{2}\right)=g+3$ il existe $i$ tel que $f_{i} \notin M$. Si $i \neq 1$ on a

$$
f_{1}+f_{i} \in L\left((g+1) V_{1}+(g+1) V_{2}\right)
$$

et

$$
\begin{aligned}
& \operatorname{ord}_{V_{j}}\left(f_{1}+f_{i}\right)=-(g+1) \quad(j=1,2), \\
& \operatorname{ord}_{u}\left(f_{1}+f_{i}\right) \geq 0 \quad \text { pour tout } u \in \Sigma-\left\{V_{1}, V_{2}\right\} .
\end{aligned}
$$


On peut donc supposer, sans restreindre la généralité, que $f_{1}$ n'est pas une combinaison linéaire de $1, h_{1}, \ldots, h_{1}^{g+1}$. La relation $(3.7)$ entraîne que $f_{1}$ est une combinaison linéaire des $1, h_{1}, \ldots, h_{1}^{g+1}$, ce qui est contradictoire. Donc les racines de $G\left(X^{\prime}\right)$ sont toutes simples.

Les coefficients $a_{0}^{\prime}, a_{1}^{\prime}, \ldots, a_{2 g+2}^{\prime}$ de $E\left(X^{\prime}\right)$ sont des éléments de $K$; on a

$$
\left|a_{0}^{\prime}\right|_{v}, \ldots,\left|a_{2 g+2}^{\prime}\right|_{v} \leq A_{v}^{36(3 g+4)(g+1) 2^{g} N^{3}} B_{v}^{2^{g+3}(g+1)(3 g+4)} v\left(2^{7(2 g+1)^{3}}\right) .
$$

Soit $V_{1}=V_{2}$. Alors le degré de la fonction $f_{1}$ est $2 g+1$ et le degré de $h_{1}$ est 2. Donc $\left[K(C): K\left(f_{1}\right)\right]=2 g+1$ et $\left[K(C): K\left(h_{1}\right)\right]=2$. On en déduit que $K(C)=K\left(h_{1}, f_{1}\right)=K\left(X^{\prime}, \Phi^{\prime}\right)$.

Soit $V_{1} \neq V_{2}$. Si $f_{1} \in K\left(h_{1}\right)$ il existe $G(T), H(T) \in K[T]$ avec $\operatorname{pgcd}(G, H)=1$ tels que $f_{1}=G\left(h_{1}\right) / H\left(h_{1}\right)$.

Comme

$$
\left[K(C): K\left(f_{1}\right)\right]=\left[K(C): K\left(h_{1}\right)\right]\left[K\left(h_{1}\right): K\left(f_{1}\right)\right]
$$

on en déduit que $\left[K\left(h_{1}\right): K\left(f_{1}\right)\right]=g+1$. Le polynôme irréductible de $h_{1}$ sur $K\left(f_{1}\right)$ est $G(T)-f_{1} H(T)$. Donc $\max \{\operatorname{deg} G, \operatorname{deg} H\}=g+1$. D'autre part, on a

$$
-(g+1)=\operatorname{ord}_{V_{j}}\left(f_{1}\right)=\operatorname{ord}_{V_{1}}\left(G\left(h_{1}\right) / H\left(h_{1}\right)\right)=-\operatorname{deg} G+\operatorname{deg} H .
$$

Alors $\operatorname{deg} H=0$. Il en résulte que $f_{1}$ est une combinaison linéaire de $1, h_{1}, \ldots, h_{1}^{g+1}$, ce qui n'est pas le cas. Donc $f_{1} \notin K\left(h_{1}\right)$ et on a $K(C)=$ $K\left(f_{1}, h_{1}\right)=K\left(X^{\prime}, \Phi^{\prime}\right)$. Par conséquent les courbes $F(X, \Phi)=0$ et $\Phi^{\prime 2}=$ $F\left(X^{\prime}\right)$ sont birationnelles sur $K$.

4. Une équation satisfaite par $X^{\prime}$ sur $K[X]$. Soient $u_{1}, \ldots, u_{r}$ les éléments de $\Sigma_{\infty}$ et $e_{1}, \ldots, e_{r}$ ses indices de ramification. Soient $X_{u_{i}}=$ $(1 / X)^{1 / e_{i}}$ et $U_{i}$ le groupe des $e_{i}$-ièmes racines de 1 . Notons

$$
\vartheta_{u_{i} \zeta}=\sum_{s=t_{0}\left(u_{i}\right)}^{\infty} \gamma_{s}\left(u_{i}\right) \zeta^{s} X_{u_{i}}^{s}
$$

où $\zeta \in U_{i}$, le développement de Puiseux de $X^{\prime}$ dans le corps des séries formelles $K\left(\left(X_{u_{i}} \zeta\right)\right)$. Alors $X^{\prime}$ est racine du polynôme

$$
P(X, T)=\prod_{\substack{u_{i} \in \Sigma_{\infty} \\ \zeta \in U_{i}}}\left(T-\vartheta_{u_{i} \zeta}\right)=T^{n}+p_{1} T^{n-1}+\ldots+p_{n}
$$

où $p_{i}$ sont les polynômes élémentaires symétriques en $\vartheta_{u_{i} \zeta}$ à signe près. Alors $p_{i} \in K(X)$. Comme les pôles de $X^{\prime}$ se trouvent à l'infini on déduit que $p_{i} \in K[X]$.

Si $V=\tau(V)$, le seul pôle de la fonction $X^{\prime}$ se trouve en $V$ et il est d'ordre 2 ; alors on a $t_{0}(V)=-2$ et $t_{0}(u)=0$ pour $u \neq V$. Si $V \neq \tau(V)$, les seuls pôles de $X^{\prime}$ se trouvent en $V$ et $\tau(V)$ et ils sont d'ordre 1; on a donc 
$t_{0}(V)=-1, t_{0}(\tau(V))=-1$ et $t_{0}(u)=0$ pour $u \neq V, \tau(V)$. Par conséquent chaque $p_{i}$ contient seulement des puissances de $(1 / X)^{\mu}$ avec $\mu \geq-2$. Donc $p_{i}$ est un polynôme quadratique en $X$. Soit

$$
p_{i}=\sum_{s=0}^{2} \pi_{i s} X^{s}=\sum_{s=-2}^{0} \pi_{i,-s}(1 / X)^{s} .
$$

On a

$$
\pi_{i,-s}=(-1)^{i} \sum_{\substack{u_{1}, \zeta_{1}, s_{1}, \ldots, u_{i}, \zeta_{i}, s_{i} \\ s_{1} / e_{1}+\ldots+s_{i} / e_{i}=s}} \gamma_{s_{1}}\left(u_{1}\right) \zeta_{1}^{s_{1}} \ldots \gamma_{s_{i}}\left(u_{i}\right) \zeta_{i}^{s_{i}}
$$

où $u_{1}, \ldots, u_{i} \in \Sigma_{\infty}, \zeta_{j} \in U_{j}$ et les paires $u_{j}, \zeta_{j}$ sont deux-à-deux distinctes.

Soit $u \in \Sigma_{\infty}$. Etudions d'abord le cas où $\tau(V)=V$. Considérons la base $\left\{f_{1}, \ldots, f_{g+2}\right\}$ de l'espace $L((2 g+1) V)$. Le théorème C.2 de [6] entraîne que le développement de Puiseux de $f_{i}$ en $u$ est

$$
f_{i}=\sum_{s=-(2 g+1)}^{\infty} b_{i s}(u) X_{u}^{s}
$$

où $b_{i s}(u)$ sont des éléments d'un corps de nombres $K(u) \supseteq K$; il existe des $K$-systèmes $\left\{A_{v}(u)\right\}$ et $\left\{B_{v}(i)\right\}$ tels que

$$
\left|b_{i s}(u)\right|_{v} \leq A_{v}(u)^{s+4 N^{3}+(g+1) n} B_{v} \quad(i=1, \ldots, g+2)
$$

où $B_{v}$ est le produit des $B_{v}(i)$; on a aussi des relations analogues à (3.2), (3.3) le développement de Puiseux de $h_{i}$ en $u$ est

$$
h_{i}=\sum_{s=-2}^{\infty} h_{i s}(u) X_{u}^{s}
$$

où $h_{i s}(u) \in K(u)$ et on a

$$
\left|h_{i s}(u)\right|_{v} \leq A_{v}(u)^{s+2^{g-1}(g+1) n+2^{g+1} N^{3}} A_{v}(V)^{2^{g-1}(g+1) n+2^{g+1} N^{3}} B_{v}^{2^{g}} v\left(2^{g}\right) .
$$

Comme $X^{\prime}=h_{2}$ pour tout $v \in M(K)$ et tout prolongement de $|\cdot|_{v}$ à $K(u)$ on a

où

$$
\left|\gamma_{s}(u)\right|_{v} \leq A_{v}(u)^{s+2^{g-2} 9 N^{3}} C_{v}
$$

$$
C_{v}=A_{v}(V)^{2^{g-2} 9 N^{3}} B_{v}^{2^{g}} v\left(2^{g}\right) .
$$

Soit $L$ un corps de nombres tel que $K\left(u_{i}\right) \subseteq L$ et $U_{i} \subseteq L(i=1, \ldots, r)$. Alors pour tout $v \in M(L)$ la valeur absolue de chacun des termes de la somme (4.2) est majorée par

$$
A_{v}\left(u_{1}\right)^{s_{1}+2^{g-2} 9 N^{3}} \ldots A_{v}\left(u_{i}\right)^{s_{i}+2^{g-2} 9 N^{3}} C_{v}^{i} .
$$

On a $s_{j} \geq-2$ si $u_{j}=V$ et $s_{j} \geq 0$ si $u_{j} \neq V$. Il en résulte que chaque partie de la somme $s_{1} / e_{1}+\ldots+s_{i} / e_{i}$ est $\geq-2$; alors $s_{j} \leq 2 e_{j} \leq 2 n$. Donc 
la valeur absolue de chacun des termes de la somme (4.2) est majorée par $C_{v}^{n} D_{v}$ où

$$
D_{v}=\prod_{j=1}^{r} A_{v}\left(u_{j}\right)^{2^{g-2} 10 N^{3} e_{j}} .
$$

Cela entraîne

$$
\left|\pi_{i s}\right|_{v} \leq C_{v}^{n} D_{v} v\left(\left(5 n^{2}\right)^{n}\right) .
$$

Considérons maintenant le cas où $\tau(V) \neq V$. Soit $\left\{f_{1}, \ldots, f_{g+3}\right\}$ la base de l'espace $L((g+1) V+(g+1) \tau(V))$. Le développement de Puiseux de $f_{i}$ en $u$ est

$$
f_{i}=\sum_{s=-g-1}^{\infty} b_{i s}(u) X_{u}^{s}
$$

où $b_{i s}(u)$ sont des éléments d'un corps de nombres $K(u) \supseteq K$. Il existe un $K$-système $\left\{A_{v}(u)\right\}$ vérifiant des relations analogues à (4.3) et (3.5). Le développement de Puiseux de $h_{i}$ en $u$ est

$$
h_{i}=\sum_{s=-1}^{\infty} h_{i s}(u) X_{u}^{s}
$$

où $h_{i s}(u) \in K(u)$ et on a

$$
\left|h_{i s}(u)\right|_{v} \leq A_{v}(u)^{s+2^{g}(g+1) n+2^{g+2} N^{3}} A_{v}^{2^{g}(g+1) n+2^{g+2} N^{3}} B_{v}^{2^{g+1}} v\left(2^{g+1}\right) .
$$

On procède comme dans le cas où $\tau(V)=V$ et on obtient

$$
\left|\pi_{i s}\right|_{v} \leq C_{v}^{n} D_{v} v\left(\left(4 n^{2}\right)^{n}\right)
$$

où

$$
C_{v}=A_{v}^{2^{g-1} 9 N^{3}} B_{v}^{2^{g+1}} v\left(2^{g+1}\right) \quad \text { et } \quad D_{v}=\prod_{j=1}^{r} A_{v}\left(u_{j}\right)^{2^{g-1} 10 N^{3} e_{j}} .
$$

5. L'équation canonique. Supposons d'abord $\tau(V)=V$. Considérons la quantité

$$
\begin{aligned}
W_{v}=A_{v}(V)^{2^{g-2} 9 N^{4}+54(g+1)(2 g+1) 2^{g-1} N^{3}} B_{v}^{2^{g} N+6(g+1)(2 g+1) 2^{g}} & \\
& \times \prod_{j=1}^{r} A_{v}\left(u_{j}\right)^{2^{g-2} 10 N^{3} e_{j}} v\left(2^{7(2 g+1)^{3}}\left(2^{g} 5 n^{2}\right)^{n}\right) .
\end{aligned}
$$

Alors $\left\{W_{v}\right\}$ est un $K$-système.

Soient $M_{\infty}(K)$ et $M_{0}(K)$ les ensembles des indices des valeurs absolues archimédiennes et non archimédiennes respectivement. Notons

$$
N_{K j}\left\{W_{v}\right\}=\prod_{v \in M_{j}(K)} W_{v}^{\delta_{v}}, \quad j=0, \infty,
$$


où $\delta_{v}$ est le degré local de $v$. Le lemme 4 de [4] entraîne qu'il existe un entier algébrique $\alpha \in K, \alpha \neq 0$, tel que

$$
|\alpha|_{v} \leq W_{v}^{-1} \quad \text { pour tout } v \in M_{0}(K)
$$

et

$$
|\alpha|_{v} \leq\left(\left|D_{K}\right|^{1 / 2} N_{K 0}\left\{W_{v}\right\}\right)^{1 / \operatorname{deg} K} \quad \text { pour tout } v \in M_{\infty}(K) .
$$

Posons

$$
X_{1}=\alpha^{2} a_{2 g+1}^{\prime} X^{\prime}, \quad \Phi_{1}=\alpha^{2 g+1} a_{2 g+1}^{\prime g} \Phi^{\prime} .
$$

Alors on a

$$
\Phi_{1}^{2}=X_{1}^{2 g+1}+a_{1} X_{1}^{2 g}+\ldots+a_{2 g+1}
$$

où

Comme

$$
a_{i}=\alpha^{2 i} a_{2 g+1-i}^{\prime} a_{2 g+1}^{\prime i-1} \quad(i=1, \ldots, 2 g+1) .
$$

$$
\left|a_{i}\right|_{v}=\left|\alpha^{2 i}\right|_{v}\left|a_{2 g+1-i}^{\prime}\right|_{v}\left|a_{2 g+1}^{\prime i-1}\right|_{v} \leq\left(W_{v}^{-1}\right)^{i}<1
$$

pour tout $v \in M_{0}(K)$ il en résulte que les $a_{i}(i=1, \ldots, 2 g+1)$ sont des entiers algébriques de $K$.

Considérons le polynôme $Q(T)=\alpha^{n} P(T / \alpha)$. Alors $Q\left(\alpha X^{\prime}\right)=0$. Les coefficients de $Q(T)$ sont des polynômes quadratiques

$$
q_{i}(X)=\alpha^{i} \pi_{i 0}+\alpha^{i} \pi_{i 1} X+\alpha^{i} \pi_{i 2} X^{2} .
$$

Alors $\left|\alpha^{i} \pi_{i s}\right|_{v} \leq 1$, pour tout $v \in M_{0}(K)$. Par conséquent $\alpha^{i} \pi_{i s} \in O_{K}$, où $O_{K}$ est l'anneau des entiers de $K$. Il en résulte que $\alpha X^{\prime}$ est un élément entier sur $O_{K}[X]$. Comme $\alpha a_{2 g+1}^{\prime}$ est un entier de $K, X_{1}$ est entier sur $O_{K}[X]$. Par conséquent si $X, \Phi$ sont des entiers de $k$, alors $X_{1}, \Phi_{1}$ sont des entiers de $K$.

Notons $f\left(X_{1}\right)=X_{1}^{2 g+1}+a_{1} X_{1}^{2 g}+\ldots+a_{2 g+1}$. Alors

$$
|f|_{v} \leq W_{v}^{2 g+1}\left(\left|D_{K}\right|^{1 / 2} N_{K 0}\left\{W_{v}\right\}\right)^{4 g+2 / \operatorname{deg} K}
$$

pour tout $v \in M_{\infty}(K)$. Comme les $a_{i}(i=1, \ldots, 2 g+1)$ sont des entiers de $K$ on a

$$
\begin{aligned}
H_{K}(f) & \leq \prod_{v \in M_{\infty}(K)}\left(W_{v}^{(2 g+1) \delta_{v}}\left(\left|D_{K}\right|^{1 / 2} N_{K 0}\left\{W_{v}\right\}\right)^{(4 g+2) \delta_{v} / \operatorname{deg} K}\right) \\
& \leq\left|D_{K}\right|^{2 g+1}\left(N_{K}\left\{W_{v}\right\}\right)^{4 g+2} .
\end{aligned}
$$

D'autre part, on a

$$
N_{K}\left\{W_{v}\right\}<c_{1}(d, N) H_{K}(F)^{6 \cdot 10^{3}(g+2) 2^{g-2} N^{15}}
$$

où

$$
c_{1}(d, N)<(3 N)^{24 \cdot 10^{3} d 2^{N^{2}} N^{18}},
$$


ce qui donne

$$
H_{K}(f)<c_{2}(d, N)\left|D_{k}\right|^{(2 g+1) N} H_{k}(F)^{2 \cdot 10^{4}(2 g+1)(g+2) 2^{g-2} N^{16}}
$$

avec

$$
c_{2}(d, N)<(3 N)^{6 \cdot 10^{4} d 2^{N^{2}} N^{20}} .
$$

Supposons maintenant $\tau(V) \neq V$. Considérons la quantité

$$
\begin{aligned}
& W_{v}=A_{v}^{2^{g-1}} 9 N^{4}+36(3 g+4)(g+1) 2^{g} N^{3} B_{v}^{2^{g+1} N+2^{g+3}}(g+1)(3 g+4) \\
& \times \prod_{j=1}^{r} A_{v}\left(u_{j}\right)^{2^{g-1} 10 N^{3} e_{j}} v\left(2^{7(2 g+1)^{3}}\left(2^{g+1} 4 n^{2}\right)^{n}\right) .
\end{aligned}
$$

Alors $\left\{W_{v}\right\}$ est un $K$-système.

Comme précédemment il existe un entier algébrique $\alpha \in K, \alpha \neq 0$, vérifiant des relations analogues à (5.1) et (5.2). Posons

$$
X_{1}=a_{2 g+2}^{\prime} \alpha^{2} X^{\prime}, \quad \Phi_{1}=\left(\sqrt{a_{2 g+2}^{\prime}}\right)^{2 g+1} \alpha^{2 g+2} \Phi^{\prime} .
$$

Alors on a

$$
\Phi_{1}^{2}=X_{1}^{2 g+2}+a_{1} X^{2 g+1}+\ldots+a_{2 g+2}
$$

où

$$
a_{i}=a_{2 g+2-i}^{\prime} \alpha^{2 i} a_{2 g+2}^{\prime i-1} \quad(i=1, \ldots, 2 g+2) .
$$

Les coefficients $a_{i}$ sont des entiers algébriques de $K$. Il résulte aussi que $X_{1}$ est entier sur $O_{K}[X]$. Donc si $X, \Phi$ sont des entiers de $k$, alors $X_{1}, \Phi_{1}$ sont des entiers de $K^{\prime}=K\left(\sqrt{a_{2 g+2}^{\prime}}\right)$.

Notons $f\left(X_{1}\right)=X_{1}^{2 g+2}+a_{1} X_{1}^{2 g+1}+\ldots+a_{2 g+2}$. Alors

$$
|f|_{v} \leq W_{v}^{2 g+2}\left(\left|D_{K}\right|^{1 / 2} N_{K 0}\left\{W_{v}\right\}\right)^{4 g+4 / \operatorname{deg} K}
$$

pour tout $v \in M_{\infty}(K)$. Comme les $a_{i}(i=0, \ldots, 2 g+2)$ sont des entiers de $K$ on a

$$
\begin{aligned}
H_{K}(f) & \leq \prod_{v \in M_{\infty}(K)}\left(W_{v}^{(2 g+2) \delta_{v}}\left(\left|D_{K}\right|^{1 / 2} N_{K 0}\left\{W_{v}\right\}\right)^{(4 g+4) \delta_{v} / \operatorname{deg} K}\right) \\
& \leq\left|D_{K}\right|^{2 g+2}\left(N_{K}\left\{W_{v}\right\}\right)^{4 g+4} .
\end{aligned}
$$

On a aussi

$$
N_{K}\left\{W_{v}\right\}<c_{3}(d, N) H_{K}(F)^{12 \cdot 10^{3} 2^{g-2}(g+3) N^{15}}
$$

où

$$
c_{3}(d, N)<(3 N)^{48 \cdot 10^{3} d 2^{N^{2}} N^{18}} .
$$

On en déduit que

$$
H_{K}(f)<c_{4}(d, N)\left|D_{k}\right|^{(2 g+2) N} H_{K}(F)^{5 \cdot 10^{4} 2^{g-2}(g+1)(g+3) N^{16}}
$$


où

$$
c_{4}(d, N)<(3 N)^{6 \cdot 10^{4} d 2^{N^{2}} N^{20}} .
$$

6. Démonstration du théorème. Nous allons utiliser le résultat suivant :

ThÉorème A. Soient $L$ un corps de nombres de degré $\lambda, B$ l'anneau des entiers de $L$ et $D_{L}$ son discriminant. Soit $f(X)$ un polynôme de $L[X]$ de degré $n$ ayant au moins trois racines d'ordre impair. Alors si $(x, y) \in B \times B$ est une solution de l'équation $Y^{2}=f(X)$ on a

$$
\max \left\{H_{L}(x), H_{L}(y)\right\}<\exp \left\{c(\lambda, n)\left(W_{1}^{n^{n}} W_{2}^{\lambda n^{n}}\right\}\right.
$$

où

et

$$
W_{1}=D_{L}^{12} H_{L}(f)^{1000 \lambda^{2}}, \quad W_{2}=\left(\log ^{*}\left|D_{L}\right|\right)^{3}\left(\log ^{*} H_{L}(f)\right)^{5}
$$

$$
c(\lambda, n)<2^{300 \lambda^{2} n^{2 n}}
$$

(pour z réel positif on note $\log ^{*} z=\max (1, \log z)$ ).

Ceci raffine un résultat de Brindza (Acta Math. Hungar. 44 (1984), 133$139)$, dans lequel la dépendance de la majoration en $\lambda, n, D_{L}$ et $H_{L}(f)$ n'est pas donné explicitement. On peut consulter [3] pour une démonstration du théorème $\mathrm{A}$.

Premier cas : $\tau(V)=V$. La fonction $X_{1}=\alpha^{2} a_{2 g+1}^{\prime} X^{\prime}$ vérifie l'équation $R(X, T)=0$, où $R(X, T)=\left(\alpha^{2} a_{2 g+1}^{\prime}\right)^{n} P\left(X, T / \alpha^{2} a_{2 g+1}^{\prime}\right)$. Les coefficients de $R(X, T)$ sont les $\pi_{i s}\left(\alpha^{2} a_{2 g+1}^{\prime}\right)^{i}$ qui sont des entiers de $K$. Comme $R(X, T)$ n'a pas de facteur indépendant de $X$, pour tout $x_{1}$ le polynôme $R^{*}(X)=R\left(X, x_{1}\right)$ est non nul de degré $\leq 2$. Alors pour tout $v \in M_{\infty}(K)$ on a

$$
\left|R^{*}\right|_{v} \leq\left(\max _{i, s}\left|\pi_{i, s}\right|_{v}\left|\alpha^{2} a_{2 g+1}^{\prime}\right|_{v}^{i}\right)\left(\max \left(1,\left|x_{1}\right|_{v}^{n}\right)\right) v(n+1) .
$$

Si $x_{1}$ est un entier de $K$ alors les coefficients de $R^{*}$ sont des entiers de $K$; on a donc

$$
H_{K}\left(R^{*}\right) \leq\left(\left|D_{K}\right|^{1 / 2} N_{K}\left\{W_{v}\right\}\right)^{2 n} H_{K}\left(x_{1}\right)^{n}(n+1)^{\operatorname{deg} K} .
$$

Les majorations (3.1) et (5.3) entraînent

$$
H_{K}\left(R^{*}\right)<c_{5}(d, N)\left|D_{k}\right|^{N^{2}} H_{k}(F)^{2 \cdot 10^{4}(g+2) 2^{g-2} N^{17}} H_{K}\left(x_{1}\right)^{n}
$$

où

$$
c_{5}(d, N)<(3 N)^{5 \cdot 10^{4} d 2^{N^{2}} N^{19}}
$$

Soit $\Omega$ l'application birationnelle de la courbe $F(X, Y)=0$ sur la courbe $\Phi_{1}^{2}=f\left(X_{1}\right)$. Soit $(x, y) \in A \times A$ un point non singulier sur $F(X, Y)=0$; alors $\Omega(x, y)=\left(x_{1}, y_{1}\right)$ et il en résulte que $R\left(x, x_{1}\right)=0$; par conséquent on 
a $R^{*}(x)=0$. Le lemme 3 de [5] entraîne que $H(x) \leq 3 H\left(R^{*}\right)$. Alors on a $\left(x_{1}, y_{1}\right) \in O_{K} \times O_{K}$ et

$$
H_{K}(x)<3^{d N} c_{5}(d, N)\left|D_{k}\right|^{N^{2}} H_{k}(F)^{2 \cdot 10^{4}(g+2) 2^{g-2} N^{17}} H_{K}\left(x_{1}\right)^{n} .
$$

D'autre part, le théorème $\mathrm{A}$ entraîne

$$
\begin{aligned}
H_{K}\left(x_{1}\right)<\exp \left\{c_{6}(d, N)\left[D_{K}^{12} H_{K}(f)^{1000 d^{2} N^{2}}\right]^{(2 g+1)^{2 g+1}}\right. \\
\left.\times\left[\left(\log ^{*}\left|D_{K}\right|\right)^{3}\left(\log ^{*} H_{K}(f)\right)^{5}\right]^{d N(2 g+1)^{2 g+1}}\right\}
\end{aligned}
$$

où

$$
c(d, N)<2^{300 d^{2} N^{2} N^{4 N^{2}}} .
$$

Alors les majorations (3.1), (5.4), (6.1) et (6.2) entraînent le résultat.

Deuxième cas : $\tau(V) \neq V$. La fonction $X_{1}=a_{2 g+2}^{\prime} \alpha^{2} X^{\prime}$ vérifie l'équation $R(X, T)=0$, où $R(X, T)=\left(\alpha^{2} a_{2 g+2}^{\prime}\right)^{n} P\left(X, T / \alpha^{2} a_{2 g+2}^{\prime}\right)$. Les coefficients de $R(X, T)$ sont les $\pi_{i s}\left(\alpha^{2} a_{2 g+2}^{\prime}\right)^{i}$ qui sont des entiers de $K$. Pour tout $x_{1}$ le polynôme $R^{*}(X)=R\left(X, x_{1}\right)$ est non nul de degré $\leq 2$. Alors si $x_{1}$ est un entier de $K$ on a

$$
H_{K}\left(R^{*}\right) \leq\left(\left|D_{K}\right|^{1 / 2} N_{K}\left\{W_{v}\right\}\right)^{2 n} H_{K}\left(x_{1}\right)^{n}(n+1)^{\operatorname{deg} K} .
$$

Les majorations (3.1) et (5.5) entraînent

$$
H_{K}\left(R^{*}\right)<c_{7}(d, N)\left|D_{k}\right|^{N^{2}} H_{k}(F)^{3 \cdot 10^{4}(g+3) 2^{g-2} N^{17}} H_{K}\left(x_{1}\right)^{n}
$$

où

$$
c_{7}(d, N)<(3 N)^{10^{5} d 2^{N^{2}} N^{19}} .
$$

Soit $(x, y) \in A \times A$ un point non singulier sur $F(x, y)=0$; alors $\Omega(x, y)=$ $\left(x_{1}, y_{1}\right)$ et il en résulte que $R\left(x, x_{1}\right)=0$; donc $R^{*}(x)=0$. De plus $\left(x_{1}, y_{1}\right) \in$ $O_{K} \times O_{K^{\prime}}$. Comme $H(x) \leq 3 H\left(R^{*}\right)$ on conclut que

$$
H_{K}(x)<3^{d N} c_{7}(d, N)\left|D_{k}\right|^{N^{2}} H_{k}(F)^{3 \cdot 10^{4}(g+3) 2^{g-2} N^{17}} H_{K}\left(x_{1}\right)^{n} .
$$

En utilisant le théorème $\mathrm{A}$ et les majorations (3.1), (5.6) et (6.3) on obtient le résultat.

Dans le cas où $(x, y)$ est un point singulier de $F(X, Y)=0$ on obtient d'une façon élémentaire une meilleure majoration.

Remerciements. Je remercie le professeur Michel Waldschmidt pour les discussions utiles que j'ai eues avec lui pendant la préparation de ce travail.

\section{Références}

[1] A. Baker and J. Coates, Integer points on curves of genus 1, Proc. Cambridge Philos. Soc. 67 (1970), 595-602. 
[2] J. Coates, Construction of rational functions on a curve, ibid. 68 (1970), 105-123.

[3] D. Poulakis, Solutions entières de l'équation $Y^{m}=f(X)$, Sém. Théorie des Nombres de Bordeaux 3 (1991), 187-199.

[4] W. M. Schmidt, Integer points on curves of genus 1, Compositio Math. 81 (1992), $33-59$.

[5] -, Eisenstein's theorem on power series expansions of algebraic functions, Acta Arith. 56 (1990), 161-179.

[6] -, Construction and estimation of bases in function fields, J. Number Theory 39 (1991), 181-224.

[7] J. P. Serre, Lectures on the Mordell-Weil Theorem, Vieweg, 1989.

[8] C. L. Siegel, Über einige Anwendungen diophantischer Approximationen, Abh. Preuss. Akad. Wiss. 1 (1929).

[9] R. Walker, Algebraic Curves, Springer, 1978.

DÉPARTEMENT DE MATHÉMATIQUES

UNIVERSITÉ DE THÉSSALONIQUE

54006 THÉSSALONIQUE, GRÈCE 